\title{
MOMENT MAPS AND DIFFEOMORPHISMS ${ }^{\dagger}$
}

\author{
S. K. DONALDSON $\ddagger$
}

Atiyah and Bott pointed out, in [1], that the curvature of a connection on a bundle over a surface can be viewed as the "momentum" corresponding to the action of the gauge group. This observation, together with various extensions, has stimulated a great deal of work and provides a conceptual framework to understand many phenomena in Yang-Mills theory. Our purpose in this paper is to explore some similar ideas in the framework of diffeomorphism groups. We begin by identifying a moment map in a rather general setting, and then see how the ideas work in some more specific situations. We hope to show that the moment map point of view is useful, both in understanding certain established results and also in suggesting new problems in geometry and analysis. While these analytical questions are the main motivation for the work, we will concentrate here on the formal aspects and will not make any serious inroads on the analysis.

\section{Identification of moment maps.}

1.1. Volume-preserving diffeomorphisms. Suppose a Lie group $G$ acts on a symplectic manifold $(\mathcal{M}, \Omega)$, preserving the symplectic form. A moment, or momentum, map for the action is a map $\mu: \mathcal{M} \rightarrow \operatorname{Lie}(G)^{*}$ with the following property. For each element $\xi$ in the Lie algebra $\operatorname{Lie}(G)$ the function $\langle\mu, \xi\rangle$ on $\mathcal{M}$ has derivative

$$
d\langle\mu, \xi\rangle=i_{X(\xi)}(\Omega)
$$

where $X(\xi)$ is the vector field on $\mathcal{M}$ defined by the infinitesimal action of $\xi$. This is to say that $\langle\mu, \xi\rangle$ is a Hamiltonian function for the 1-parameter subgroup generated by $\xi$.

Now consider the following set-up. Let $S$ be a compact $k$-manifold with a fixed volume form $\sigma \in \Omega^{k}(S)$. Let $(M, \omega)$ be a symplectic manifold and let $\mathcal{M}$ be the infinite-dimensional space of smooth maps from $S$ to $M$, in some fixed homotopy class. This may be considered as an infinite-dimensional manifold in the usual way: the tangent space to $\mathcal{M}$ at a map $f: S \rightarrow M$ is the space of sections of the bundle $f^{*}(T M$ ) over $S$. (We will ignore, in this paper, any foundational questions about infinite dimensional manifolds.) Now $\mathcal{M}$ carries a natural symplectic form $\Omega$ : for sections $v, w$ of $f^{*}(T M)$ we define

$$
\Omega(v, w)=\int_{S} \omega(v, w) \sigma
$$

The Lie group $\mathcal{G}$ of volume-preserving diffeomorphisms of $S$ acts on $\mathcal{M}$ by composition on the right, preserving $\Omega$, so we may seek a moment map for this action. Let us suppose first that $f^{*}([\omega])$ is zero in the de Rham cohomology $H^{2}(S)$ and that $H^{1}(S)=$ 0 . Then for each $f \in \mathcal{M}$ we can make the following construction. We can, by hypothesis, choose a 1-form $a \in \Omega^{1}(S)$ such that $f^{*}(\omega)=d a$. Then for any vector field $\xi$ on $S$ we can define a pairing

$$
\langle a, \xi\rangle=\int_{S} a(\xi) \sigma
$$

\footnotetext{
$\dagger$ Received February 28, 1999; accepted for publication March 18, 1999.

$\ddagger$ Department of Mathematics, Stanford University, Stanford, CA 94305, U.S.A. (simon@math. stanford.edu).The research is supported in part by NSF grant DMS 98-03192.
} 
Suppose that $\xi$ is in the Lie algebra of $\mathcal{G}$ : that is,

$$
L_{\xi}(\sigma)=d\left(i_{\xi}(\sigma)\right)=0
$$

(here $L_{\xi}$ denotes the Lie derivative). Then the pairing $\langle a, \xi\rangle$ is independent of the choice of $a$. For, since $H^{1}(S)=0$, any other choice $a^{\prime}$ differs by the derivative of a function, $a^{\prime}-a=d g$ say, and

$$
\int_{S}(d g)(\xi) \sigma=\int_{S} d g \wedge\left(i_{\xi}(\sigma)=\int_{S} d\left(g i_{\xi}(\sigma)\right)-g d\left(i_{\xi}(\sigma)\right)=0 .\right.
$$

So in sum we have a well-defined linear map $\xi \mapsto\langle a, \xi\rangle$ on the Lie algebra of $\mathcal{G}$ which we denote by $\mu(f)$. Another way of expressing this is to say that the Lie algebra of $\mathcal{G}$ is identified with ker $d: \Omega^{k-1}(S) \rightarrow \Omega^{k}(S)$ and we have a dual pairing of this space and coker $d: \Omega^{0}(S) \rightarrow \Omega^{1}(S)$ which is isomorphic to the space ker $d: \Omega^{2}(S) \rightarrow \Omega^{3}(S)$ in which $f^{*}(\omega)$ lives.

Proposition 1. If $H^{1}(S)=0$ and $[\omega]=0$ in $H^{2}(S)$ then the above construction gives a map $\mu: \mathcal{M} \rightarrow \operatorname{Lie}(\mathcal{G})^{*}$ which is a moment map for the action of $\mathcal{G}$ on $(\mathcal{M}, \Omega)$.

To verify this, consider a map $f: S \rightarrow M$, a section $v$ of $f^{*}(T M)$ over $S$ and a volume-preserving vector field $\xi$ on $S$. Then, with $\mu$ defined as above, $\langle\mu, \xi\rangle$ is a function on $\mathcal{M}$ and $d\langle\mu, \xi\rangle$ is a 1 -form on $\mathcal{M}$, which we can evaluate on the tangent vector $v \in T \mathcal{M}_{f}$. This is the derivative $\frac{d}{d t} \mu\left(f_{t}\right)(\xi)$, where $f_{t}$ is a 1-parameter family of maps from $S$ to $M$ with $f_{0}=f$ and $t$-derivative $v$ (all time- derivatives being evaluated at $t=0)$. Now

$$
\frac{d}{d t} f_{t}^{*}(\omega)=d\left(i_{V}(\omega)\right)
$$

so $\frac{d}{d t} a_{t}=i_{V}(\omega)$ and

$$
\frac{d}{d t} \mu\left(f_{t}\right)(\xi)=\int_{S} i_{v}(\omega)\left(f_{*}(\xi) \sigma=\int_{S} \omega\left(v, f_{*}(\xi)\right)=\Omega(v, X(\xi)),\right.
$$

and this is precisely the identity required for a moment map.

We may vary the topological hypotheses in force above somewhat. First, if $f^{*}([\omega]) \neq 0$ in $H^{2}(S)$ we may fix a reference form $\nu$ in the de Rham cohomology class and choose $a$ so that $f^{*}(\omega)-\nu=d a$. The calculation goes through as before and we do get a moment map, but not an equivariant moment map, with respect to the co-adjoint action. If $H^{1}(S) \neq 0$ we may proceed in two ways. On the one hand there is a Calabi homomorphism $C$ from the group of volume-preserving diffeomorphisms to the torus $H^{n-1}(S ; \mathbf{R}) / H^{n-1}(S ; \mathbf{Z})$. The kernel of $C$ is a Lie group $\mathcal{G}_{0}$ whose Lie algebra can be identified with the exact $(n-1)$-forms, and our construction yields a moment map for the action of this group. On the other hand, suppose that the class $[\omega / 2 \pi]$ is an integral class in $H^{2}(M)$ so there is a unitary line bundle $L$ over $M$ having a connection with curvature $-i \omega$. Suppose also that $f^{*}(L)$ is trivial as a complex line bundle over $S$. Let $\tilde{\mathcal{M}}$ be the covering space of $\mathcal{M}$ consisting of pairs $(f, \tau)$ where $\tau$ is a homotopy class of trivialisations of $f^{*}(L)$, a Galois covering with group $H^{1}(S ; \mathbf{Z})$. Then for each point of $\tilde{\mathcal{M}}$ we can choose a trivialisation of $f^{*}(L)$ within the given homotopy class. The connection form in this trivialisation yields a 1-form $a$ with $d a=f^{*}(\omega)$ and our construction gives a moment map for the natural action of $\mathcal{G}$ on $\tilde{\mathcal{M}}$.

1.2. The symplectic case. Now consider the case when $S$ is also a symplectic manifold of dimension $k=2 l$, with symplectic form $\rho$. This gives rise to a volume 
form $\sigma=\rho^{l} / l$ ! and so fits into the framework above, but we can write the relevant moment map in a different way. The group of interest is now the group $\mathcal{G}^{\mathrm{Sp}}$ of symplectomorphisms of $S$.

For simplicity let us again assume that $H^{1}(S)=0$, so all symplectic vector fields on $S$ are Hamiltonian and the Lie algebra of the symplectomorphism group can be identified with the functions on $S$ modulo the constants, or equally with the functions of integral zero. This carries an invariant $L^{2}$ inner product which embeds the Lie algebra naturally in its dual. Now let $F$ be a function on $S$ and $\xi_{F}$ be the Hamiltonian vector field which it generates. Following the notation of the previous section, if $f^{*}(\omega)=d a$ the pairing $\left\langle a, \xi_{F}\right\rangle$ can be written

$$
\begin{aligned}
\left\langle a, \xi_{F}\right\rangle & =1 / l ! \int_{S} a\left(\xi_{F}\right) \rho^{l} \\
& =1 / l ! \int_{S} a \wedge d F \wedge \rho^{l-1}, \\
& =1 / l ! \int_{S} F d a \wedge \rho^{l-1} \\
& =1 / l ! \int_{S} F f^{*}(\omega) \wedge \rho^{l-1} \\
& =\int_{S} F H_{f} \sigma=\left\langle F, H_{f}\right\rangle,
\end{aligned}
$$

where $H_{f}$ is the function on $S$ defined by

$$
H_{f} \sigma=f^{*}(\omega) \wedge \rho^{l-1} .
$$

This means that the moment map for the action of $\mathcal{G}^{\mathrm{Sp}}$ is given simply by $\mu(f)=H_{f}$. Notice that $H_{f}$ is defined locally, without any topological assumptions. In the case when $H^{1}(S) \neq 0$ the relevant group consists of the exact symplectomorphisms: the kernel of the restriction of the Calabi map. To sum up we have

Proposition 3. The map $f \mapsto H_{f}$ is an equivariant moment map for the action of the group of exact symplectomorphisms of $S$ on $\mathcal{M}$.

2. Brief recap of moment map geometry. We will quickly recall some standard constructions, as background for the rest of the paper. For details we refer to [7],[8]. In this exposition we have in mind primarily the case of finite-dimensional manifolds, although of course all the formal aspects will go over immediately to infinitedimensional situations, of the kind we are considering in the body of this paper.

2.1. If $\mu$ is an equivariant moment map for the action of $G$ on $(\mathcal{M}, \Omega)$ one may form the symplectic quotient

$$
\mathcal{M} / / G=\mu^{-1}(0) / G .
$$

This is a manifold if $G$ acts freely on $\mathcal{M}$ and it inherits an induced symplectic form. Suppose now that $\mathcal{M}$ is a Kahler manifold, and $\Omega$ is the Kahler form. Suppose that the action of $G$ extends to an action of the complexified group $G^{c}$. Then, at least on an open set of "stable points", one has an identification

$$
\mathcal{M} / / G=\mathcal{M} / G^{c} .
$$

This says that on an open set of stable points, each $G^{c}$-orbit meets the zero-set $\mu^{-1}(0)$ in a unique $G$-orbit. Using this identification, one sees that the symplectic quotient 
has a natural Kahler structure.

2.2. The relation between the symplectic and complex quotients, and the role of stability, is clarified by a flow that one can define on $\mathcal{M}$ in this Kahler situation, provided that the Lie algebra of $G$ has an invariant inner product. This means that the moment map can be regarded as a map into the Lie algebra, rather than its dual. This flow is defined by the equation

$$
\frac{d x}{d t}=I X(\mu(x))
$$

for $x(t) \in \mathcal{M}$. Here $I$ denotes the usual action of complex multiplication on tangent vectors in $\mathcal{M}$. This is the gradient flow of the function $\|\mu\|^{2}$ on $\mathcal{M}$, one has:

$$
\frac{d}{d t}\|\mu(x)\|^{2}=-\|X(\mu(x))\|^{2} .
$$

This gradient flow clearly preserves the $G^{c}$-orbits in $\mathcal{M}$. The stable points, which one expects will form a dense open set, are those which flow down to the minimum of the function: the zero set of $\mu$. More generally one can study the stratification of $\mathcal{M}$ defined by infimum of $\|\mu\|^{2}$ on the $G^{c}$ orbits.

2.3. Now consider the case when $\mathcal{M}$ is a hyperkahler manifold: so we have three complex structures $I, J, K$ satisfying the algebraic relations of the quaternions and corresponding Kahler forms $\Omega_{1}, \Omega_{2}, \Omega_{3}$ defining the same Riemannian metric on $\mathcal{M}$. Suppose that the action of $G$ preserves all this structure and we have equivariant moment maps $\mu_{1}, \mu_{2}, \mu_{3}$ for the three symplectic forms. We can put these together into a single map

$$
\underline{\mu}: \mathcal{M} \rightarrow \operatorname{Lie}(G)^{*} \otimes \mathbf{R}^{3} .
$$

The hyperkahler quotient [7] is $\underline{\mu}^{-1}(0) / G$ and this inherits a hyperkahler structure. The final fact we wish to record is less well-known: it is essentially implicit in the work of Taubes [11], who studied the case of Yang-Mills theory over $\mathbf{R}^{4}$ where the hyperkahler quotient of the space of all connections is the instanton moduli space. In general, suppose that the Lie algebra of $G$ has an invariant inner product and consider the $G$-invariant function $E=\|\underline{\mu}\|^{2}$ on $\mathcal{M}$. The gradient flow of this function is given by

$$
\frac{d x}{d t}=I X\left(\mu_{1}(x)\right)+J X\left(\mu_{2}(x)\right)+K X\left(\mu_{3}(x)\right)
$$

The minimum of $E$ is obviously given by the zero-set of $\mu$, and we want to focus now on the other critical points. We associate to the problem an index $d$ equal to the dimension of the hyperkahler quotient, if this is nonempty. Thus, in finite dimensions:

$$
d=\operatorname{dim}(\mathcal{M})-4 \operatorname{dim} G \text {. }
$$

In suitable infinite-dimensional problems one interprets this as minus the Fredholm index of a linear operator $L$. Given any point $x \in \mathcal{M}$ the infinitesimal action is a linear $\operatorname{map} \xi \mapsto X_{x}(\xi)$ from $\operatorname{Lie}(G)$ to $T \mathcal{M}_{x}$. The operator $L=L_{x}: \mathbf{H} \otimes \operatorname{Lie} G \rightarrow T \mathcal{M}_{x}$ is formed from this using the action of the quaternions:

$$
L\left(\xi_{0}+i \xi_{1}+j \xi_{2}+k \xi_{3}\right)=X_{x}\left(\xi_{0}\right)+I X_{x}\left(\xi_{1}\right)+J X_{x}\left(\xi_{2}\right)+K X_{x}\left(\xi_{3}\right) .
$$

If $\mathcal{M}$ is connected the index of $L_{x}$ will not depend upon $x$. If $x$ lies in the zero set of $\mu$ the tangent space of the hyperkahler quotient at $x$ can be identified (modulo a suitable implicit function theorem) with the kernel of the adjoint of $L_{x}$. 
Proposition 6. If $d \geq 0$ then there are no strictly stable critical points of $E$ on $\mathcal{M}$ outside the minimum set $\mu^{-1}(0)$.

We outline a proof of this proposition. The equation defining a critical point $x$ of $E$ is

$$
L_{x}(\underline{\mu}(x))=0 .
$$

So if $\mu(x) \neq 0, L_{x}$ has a non-trivial kernel. The hypothesis $d \geq 0$ then imples that the adjoint operator $L^{*}$ has a non-trivial kernel. The operator is $\mathbf{H}$-linear, so the kernel is a quaternionic vector space. Let $v$ be an element of this kernel and $H$ be the Hessian of $E$ at $x$, a quadratic form on $T \mathcal{M}_{x}$. A calculation shows that

$$
H(v)+H(I v)+H(J v)+H(K v)=0 .
$$

So $H$ cannot be positive definite, as asserted.

One can hope, at least in particular cases, to strengthen this statement to show that $H$ has a non-trivial negative subspace and further to get a lower bound on the dimension, as in the work of Taubes.

\section{Moduli spaces.}

3.1. Special Lagrangian submanifolds. Suppose $M$ is a complex $n$-manifold with a non-vanishing holomorphic $n$ - form $\theta \in \Omega^{n, 0}(M)$. Suppose that in addition $M$ has a Kahler metric $\omega$. Then a special Lagrangian submanifold $P \subset M$ is, by definition, a submanifold of (real) dimension $n$ such that

(1) the restriction of $\theta$ to $P$ is a real $n$-form:

(2) the restriction of $\omega$ to $P$ is zero, i.e. $P$ is a Lagrangian submanifold in the ordinary sense of symplectic geometry.

These submanifolds were introduced by Harvey and Lawson [5] and have been studied intensively, following the work of Strominger, Yau and Zaslow [10], in the context of mirror symmetry [4],[6]. They may be fitted into our general picture as follows. Consider a fixed compact $n$-manifold $S$ with a volume form $\sigma$, and suppose first that $H^{1}(S)=0$. Then the group of volume-preserving diffeomorphisms of $S$ acts on the space $\mathcal{M}$ of maps from $S$ to $M$ in a given homotopy class and we have identified a moment map for the action above. A map $f: S \rightarrow M$ is a zero of the moment map precisely when $f^{*}(\omega)=0$. Now the complex structure on $M$ means that we can regard $\mathcal{M}$ as an infinite dimensional complex manifold, via the complex structure on the bundles $f^{*}(T M)$, moreover $\Omega$ becomes a Kahler form on $\mathcal{M}$. Consider now the subset $\mathcal{N} \subset \mathcal{M}$ consisting of maps $f: S \rightarrow M$ with $f^{*}(\theta)=\sigma$. Note that such maps are necessarily immersions. This subset $\mathcal{N}$ is clearly preserved by the action of $\mathcal{G}$ on $\mathcal{M}$. Moreover $\mathcal{N}$ is a complex submanifold of $\mathcal{M}$. For, by definition, it is the zero-set of the map $f \mapsto f^{*}(\theta)-\sigma$, which we can regard as mapping to the vector space of complex $n$-forms on $S$ of integral 0 . The derivative of this map, at a point $f_{0} \in \mathcal{N}$, is the linear map $D$,

$$
D(v)=L_{v}(\theta)=d\left(i_{v}(\theta)\right)
$$

for $v \in T \mathcal{M}_{f_{0}}=\Gamma\left(f^{*}(T M)\right)$. Here we have used the fact that $d \theta=\bar{\partial} \theta=0$. Now $D$ maps onto the forms of integral zero, so $\mathcal{N}$ is a submanifold, and moreover $D$ is complex linear, since $\theta$ has type $(n, 0)$, so $\mathcal{N}$ is a complex submanifold. The zeros of the moment map for the action of $\mathcal{G}$ on $\mathcal{N}$ are the "parametrised" immersed special Lagrangian submanifolds of the given topological type, and the moduli space of special Lagrangian submanifolds appears in this framework as the symplectic quotient $\mathcal{N} / / \mathcal{G}$. 
As it stands the discussion above misses the cases of most interest, because if $H^{1}(S)=0$ the special Lagrangian submanifolds are isolated, and the moduli space is just a discrete set. We can extend the set-up in the manner of 1.1 to allow nontrivial $H^{1}$ using the kernel $\mathcal{G}_{0}$ of the Calabi map. The symplectic quotient $\mathcal{N} / / \mathcal{G}_{0}$ is a torus bundle over the moduli space, $V$ say, of special Lagrangian submanifolds, with fibre $T=H_{1}(S ; \mathbf{R}) / H_{1}(S ; \mathbf{Z})$. The general moment map theory yields a Kahler metric on $\mathcal{N} / / \mathcal{G}_{0}$. Unfortunately this is not quite the same as the space considered in the Mirror symmetry literature, which is a bundle over $V$ with fibre the dual torus $T^{*}=H^{1}(S ; \mathbf{R}) / H^{1}(S ; \mathbf{Z})$. (However Hitchin.has shown how to modify the construction to fit in with the literature on the geometry of this latter space). In the special case when $n=2$, which we will discuss further below, the two spaces are the same since $T$ is then isomorphic to $T^{*}$.

3.2. The symplectic case. There is a parallel discussion in the case when $M$ is a complex symplectic manifold, so there is a holomorphic symplectic form $\Theta \in$ $H^{2,0}(M)$. We call an (immersed) submanifold $P \subset M$ (with $\operatorname{dim}_{\mathbf{R}} P=\operatorname{dim}_{\mathbf{C}} M=n$ ) anLS-submanifold if it is Lagrangian with respect to the (real) symplectic form $\operatorname{Re}(\Theta)$ and symplectic with respect to the (real) symplectic form $\operatorname{Im}(\Theta)$. (Of course we can always replace $\Theta$ by $i \Theta$, reversing the roles of the real and imaginary parts.) We digress to point out the following example:

EXAmPle 7 . Let $Z$ be any complex manifold with $H^{1}(Z)=0$ and let $M$ be the total space of the cotangent bundle $T^{*} Z$, with the canonical complex structure and holomorphic symplectic form $\Theta$. We consider those LS submanifolds $P \subset T^{*} Z$ which are graphs of sections of the fibration $T^{*} Z \rightarrow Z$. The real part of $\Theta$ is the canonical 2-form on $T^{*} Z$, regarded as the real cotangent bundle, so the first condition says that $P$ is the graph of an exact 1 -form $d \phi$, where $\phi$ is a real-valued function on $Z$. A litle thought shows that second condition asserts that $i \bar{\partial} \partial \phi>0$ on $Z$, i.e. $\phi$ is a Kahler potential. Thus the LS-graphs in $T^{*} Z$ can be identified with the Kahler potentials modulo constants. There is a global variant of this which applies to any complex manifold $Z$ with a holomorphic line bundle $L \rightarrow Z$. Let $p: J_{1}(L) \rightarrow Z$ be the bundle of 1-jets of sections of $L$ and let $U \subset J_{1}(L)$ be the subset of jets of non-vanishing sections. Let $M_{L}$ be the fibrewise quotient of $U$ by the natural action of $\mathrm{C}^{*}$. Locally in $Z$ we can trivialise $L$ which identifies $M_{L}$ with the cotangent bundle, and two different trivialisations induce the same 2 -form on $M_{L}$, so $M_{L}$ has a canonical complex symplectic structure. Then the LS-sections of $M_{L}$ can be identified with the Kahler metrics on $M$ in the cohomology class $c_{1}(L)$.

Returning to the main theme, fix a real symplectic manifold $(S, \rho)$ and consider the set $\mathcal{N}$ of maps $f: S \rightarrow M$ with $f^{*}(\Theta)=i \rho$. Then, just as in the previous case, $\mathcal{N}$ is an infinite-dimensional complex manifold and the group $\mathcal{G}_{0}^{\mathrm{Sp}}$ of exact symplectomorphisms of $S$ acts on $\mathcal{N}$. Now suppose that $M$ has in addition a Kahler form $\omega$. So we have three different symplectic forms on $M: \omega_{1}=\operatorname{Re}(\Theta), \omega_{2}=\operatorname{Im}(\Theta), \omega_{3}=\omega$. Then $\mathcal{N}$ gets an induced Kahler structure and the Kahler quotient $\mathcal{N} / / \mathcal{G}_{0}^{\mathrm{Sp}}$ is a torus bundle over the moduli space of submanifolds $P \subset M$ which satisfy the three conditions

$$
\left.\omega_{1}\right|_{P}=0,\left.\omega_{2}\right|_{P} \text { is nondegenerate },\left.\omega_{3}\right|_{P} \wedge \omega_{2}^{n-1}=c \omega_{2}^{n} .
$$

Here $c$ is a constant determined by the homotopy class of the map, which we are allowed to include since the constants act trivially as Hamiltonians. The general theory tells us that this torus bundle over the moduli space inherits a natural Kahler structure. 
3.3. The hyperkahler case. In the case when $M$ has real dimension 4 the two discussions co-incide. It is natural also then to suppose that $M$ is a hyperkahler 4-manifold, with three complex structures $I, J, K$ giving an action of the quaternions, and three Kahler forms $\omega_{1}, \omega_{2}, \omega_{3}$, with a symmetry under the group $S O(3)$. From either point of view the objects we are studying are, after a suitable rotation of the complex structures, complex curves in $M$. We obtain then

Proposition 8. Suppose $M$ is a hyperkahler 4-manifold and let $\alpha$ be a homology class in $H_{2}(M ; \mathbf{Z})$. Suppose that $\omega_{2}(\alpha)=\omega_{3}(\alpha)=0$ and $\omega_{1}(\alpha)>0$. There is a hyperkahler metric on the moduli space of pairs $(C, \lambda)$ where $C$ is a smooth $I$ holomorphic curve in the homology class $\alpha$ and $\lambda$ is a holomorphic line bundle of degree 0 over $C$.

This structure arises because the moduli space can be regarded as the hyperkahler quotient of $\mathcal{M}$ by the group $\mathcal{G}_{0}^{\mathrm{Sp}}$.

4. Minimising the norm of the moment map. Suppose again that $S$ is a symplectic manifold and $M$ is Kahler. Then we are in the familar formal picture with a mapping space $\mathcal{M}$ which is Kahler and a symmetry group $\mathcal{G}^{\mathrm{Sp}}$ whose Lie algebra admits an invariant inner product-the $L^{2}$ norm. So we may ask how the circle of ideas sketched in II.2 works in this context. On the one hand we may try to identify "orbits" of the complexification and search for zeros of the moment map in these orbits. On the other hand we can look at the gradient flow (4) of the norm of the moment map. It is not clear whether this programme is sensible in general: for example the gradient flow equation is not usually parabolic and one cannot be sure if solutions exist even for a short time with smooth initial data. In this section we will examine a number of cases when we do arrive at apparently sensible differential geometric problems.

4.1. Diffeomorphisms of surfaces. Suppose here that $M$ is a compact Riemann surface with a fixed metric $\omega$, and that $S$ is diffeomorphic to $M$. There is no loss in supposing that the total areas of $M, S$ are equal. Restrict attention to the open set in $\mathcal{M}$ of oriented diffeomorphisms $f: S \rightarrow M$. Any such $f$ defines an area form $\left(f^{*}\right)^{-1}(\rho)$ on the Riemann surface $M$ which we can write as $J_{f} \omega$, where $J_{f}$ is a positive function on $S$. By definition, $J_{f}$ determines $f$ up to the action of $\mathcal{G}^{\text {Sp }}$. The gradient flow equation is

$$
\frac{d}{d t} f_{t}=I f_{*}\left(\xi_{H\left(f_{t}\right)}\right)
$$

where $H(f)$ is the moment map-the function $f^{*}(\omega) / \rho$ on $S$-and $\xi_{H}$ is the Hamiltonian vector field of $H$. Thus $H(f)$ is the composite of $f$ with the real-valued function $J_{f}^{-1}$ on $S$. The evolution equation can be written as an evolution equation for $J_{f}$. For the image of the vector field $\xi_{H}$ under $f_{*}$ is $\operatorname{Igrad}\left(J_{f}^{-1}\right)$ so

$$
\frac{d}{d t} J_{f_{t}}=\operatorname{div}\left(I f_{*}\left(\xi_{H\left(f_{t}\right)}\right)\right)=\operatorname{divgrad}\left(J_{f}^{-1}\right),
$$

and $J(t)=J_{f_{t}}$ satisfies the equation

$$
\frac{d}{d t} J=\Delta\left(J^{-1}\right)
$$

where $\Delta$ is the ordinary Laplace operator on $M$. Conversely, given a positive solution $J(t)$ of this equation (10), with $J(0)=J_{f_{0}}$, we can define a time-dependent vector 
field on $M$,

$$
X_{t}=\operatorname{grad}(J(t))
$$

and let $\psi_{t}: M \rightarrow M$ be the family of diffeomorphisms obtained from the integral curves of $X_{t}$. Then the composites $f_{t}=\psi_{t} \circ f_{0}$ satisfy (9). Now equation (10) is parabolic and the maximimum principle implies that $\max (J)$ is decreasing and $\min (J)$ is increasing. It is a straightforward exercise to show that, with any initial data, solutions exist for all time and converge to constant functions. But maps with $J_{f}$ constant are just the area-preserving maps from $S$ to $M$ which are precisely the zeros of the moment map (since the cosntants act trivially) - just what we would expect in the general picture.

4.2. The reverse porous-medium equation. Here we ask what happens if we vary the set-up above to allow maps which are not diffeomorphisms. Consider the case when $M=\mathbf{C}$, the complex plane, with its standard symplectic structure, and let $S$ be a compact surface with area form $\rho$. A map $f: S \rightarrow \mathbf{C}$ can be written as $f_{1}+i f_{2}$ for real valued functions $f_{1}, f_{2}$ on $S$. We can also think of these maps as elements of the complexification of the Lie algebra of functions on $S$ under Poisson bracket, and the equation (9) becomes

$$
\begin{aligned}
& \frac{d}{d t} f_{1}=\left\{\left\{f_{1}, f_{2}\right\}, f_{2}\right\} \\
& \frac{d}{d t} f_{2}=\left\{\left\{f_{2}, f_{1}\right\}, f_{1}\right\}
\end{aligned}
$$

(Note that we may study the corresponding ODE for pairs of elements of any Lie algebra.) The zeros of the moment map are pairs $\left(f_{1}, f_{2}\right)$ with $\left\{f_{1}, f_{2}\right\}=0$, and these are just the maps which have a 1-dimensional image in $\mathbf{C}$. Suppose that $S$ is the double of a manifold with bounclary $S_{+}$and so has a fixed involution $\sigma: S \rightarrow S$. Assume this is compatible with the symplectic form, so $\sigma^{*}(\rho)=-\rho$. Restrict attention to the set $\mathcal{U}$ of maps $f: S \rightarrow \mathbf{C}$ with $f \circ \sigma=f$ and such that $f$ maps the interior of $S_{+}$ diffeomorphically to its image in $\mathbf{C}$, a domain $\Omega_{f} \subset \mathbf{C}$. We can define a function $J_{f}$ on $\Omega_{f}$ in the same fashion as in (4.1), using the restriction of $f$ to $\Sigma_{+}$. The equation (11) corresponds to the same equation (10) in the interior of $\Omega_{f}$, but this must be supplemented by boundary conditions. Since the domain $\Omega_{f}$ depends upon $f$ we encounter a "free boundary problem". One can show that the appropriate equation is just (10) where $J^{-1}$ is viewed as a distribution on C-extended by zero outside $\Omega_{f}$. Now the function $J_{f}$ is typically unbounded: for a generic map $f$ of the kind we are considering $J_{f}=O\left(d^{-1 / 2}\right)$ where $d$ is the distance to the boundary of $\Omega_{f}$. If we write $U=J_{f}^{-2}$ then, generically at least, $U$ is smooth up to the boundary and vanishes transversally there. Our equation can now be written in terms of $U$, and becomes

$$
\frac{d U}{d t}=-U \Delta U-\frac{1}{2}|\nabla U|^{2}
$$

where we have in mind that $U_{t}$ is a positive function on a domain $\Omega_{t}$, vanishing on the boundary, and the evolution of $\Omega_{t}$ is determined by saying that the boundary moves inwards with normal velocity $|\nabla U|$. This can be compared with the porous medium equation, much studied in applied mathematics, which is

$$
\frac{d U}{d t}=-U \Delta U+\frac{1}{2}|\nabla U|^{2} .
$$


In this latter case the domain expands; the boundary moving outwards with normal velocity $|\nabla U|$.

Now, modulo questions of smoothness on the boundary, if we are given a solution of (12) we can recover a solution of (11) by integrating a time-dependent vector field just as before. The zeros of the moment map do not lie inside the open set $\mathcal{U}$ we are considering at present but clearly there are sequences $f_{i}$ of maps in $\mathcal{U}$ which converge to zeros of the moment map. In terms of the functions $J_{f}$ this corresponds to $J_{f_{i}} \rightarrow \Gamma$ as measures on $\mathbf{C}$, where $\Gamma$ is a measure supported on a 1-dimensional set $|\Gamma| \subset \mathbf{C}$. So we are lead to propose the following

Problem 13. For which initial data $U_{0}$ is there a solution of the the freeboundary problem (12) defined for $t \in[0, \infty)$, such that $U_{t}^{-1 / 2}$ converges to a measure supported on a 1-dimensional subset of $\mathbf{C}$, as $t \rightarrow \infty$ ?

If such a solution does exist we interpret it as an integral curve of the gradient equation (4), converging to a zero of the moment map.

For some initial data this question does have an affirmative answer. Let $S$ be the standard 2-sphere in $\mathbf{R}^{3}$ with the induced area form, and let $x, y, z$ be the standard Euclidean co-ordinates, so

$$
\{x, y\}=z \text { etc. }
$$

generating a copy of the Lie algebra $\mathfrak{s o}(3)=\mathfrak{s u}(2)$ inside $C^{\infty}(S)$, and we can look for solution of (11) inside $\mathfrak{s o}(3)$. The simplest solution is to take:

$$
f_{1}=(2 t)^{-1 / 2} x, \quad f_{2}=(2 t)^{-1 / 2} y
$$

which yields a solution of (12) with $U_{t}$ supported on a disc of radius $(2 t)^{-1 / 2}$, and given in this disc by:

$$
U(w, t)=(2 t)^{-1}\left((4 t)^{-1}-|w|^{2}\right) .
$$

In this case the map $f_{t}$ converges to zero, and $U^{-1 / 2}$ converges to $4 \pi \delta_{0}$. More generally we have solutions:

$$
f_{1}=(\tanh (t / 2)+\operatorname{coth}(t / 2)) x, f_{2}=(\tanh (t / 2)-\operatorname{coth}(t / 2)) y
$$

which yields functions $U_{t}$ supported on ellipses and with $U_{t}^{-1 / 2}$ converging to a measure supported on the segment $[-2,2] \subset$ C. One can also show, by reducing the equation to a linear, parabolic, equation, that (13) has an affirmative answer for any circularly symmetric initial data.

However we cannot expect to find these solutions for all initial data. To see this let $G$ be the usual Green's operator on $\mathbf{C}=\mathbf{R}^{2}$

$$
G(\phi)(w)=(2 \pi)^{-1} \int_{\mathbf{R}^{2}} \phi\left(w^{\prime}\right) \log \left|w-w^{\prime}\right| d w^{\prime} .
$$

Then the equation (10) implies that

$$
\frac{d}{d t} G\left(J_{t}\right)=J^{-1}
$$

in particular $G\left(J_{t}\right)$ is non-decreasing everywhere and is constant outside the support of $J_{0}$. Suppose (13) has an affirmative answer for initial data $U_{0}$, with support $\Omega_{0}$. The restriction of $G\left(U_{0}^{-1 / 2}\right)$ is a harmonic function $h_{0}$ on $\mathbf{C} \backslash \Omega_{0}$ and this extends to a harmonic function $h_{\infty}$-the restriction of $G(\Gamma)$ - on $\mathbf{C} \backslash|\Gamma|$. Moreover $h_{\infty} \geq G\left(U_{0}^{-1 / 2}\right)$ on its domain $\mathbf{C} \backslash|\Gamma|$. One can construct examples of initial data where the function 
$h_{0}$ cannot be extended in this way, and other examples when the harmonic extension is not bounded below by $G\left(U_{0}^{-1 / 2}\right)$, so for these examples the question has a negative answer. A possibility is that these constraints on $G\left(U_{0}^{-1 / 2}\right)$ are both necessary and sufficient conditions for a positive answer to (13).

4.3. Kahler metrics. Now suppose that $M$ is a compact Kahler manifold of complex dimension $n$ and that the symplectic manifold $S$ is diffeomorphic to $M$. We restrict attention to the open set of diffeomorphisms $f: S \rightarrow M$, so the case $n=1$ was the topic of (4.1). As in that case, we can rewrite the gradient flow equation as an equation for the 1-parameter family of symplectic forms $\chi_{t}=\left(f_{t}^{*}\right)^{-1}(\rho)$ on $M$. The equation is

$$
\frac{d}{d t} \chi_{t}=L_{I \xi_{t}} \chi_{t}
$$

where $\xi_{t}$ is the Hamiltonian vector field of the function $H_{t}$ defined by

$$
H_{t} \chi^{n}=\omega \wedge \chi^{n-1}
$$

with respect to the symplectic form $\chi_{t}$. The case we wish to discuss now is when the initial data $\chi_{0}$ is a positive form of type $(1,1)$-that is, a second Kahler structure on the complex manifold $M$. In this case the quantity $L_{I \xi_{0}} \chi_{0}$ can be written as $i \bar{\partial} \partial H_{0}$ so the $t$-derivative of $\chi_{t}$ is again of type $(1,1)$. That is, the set of Kahler forms is preserved by the gradient flow. The gradient equation can now be written as an equation for a Kahler potential,

$$
\chi_{t}=\chi_{0}+i \bar{\partial} \partial \phi_{t}
$$

and becomes

$$
\frac{d \phi_{t}}{d t}=H_{t}=\frac{\omega \wedge\left(\chi_{0}+i \bar{\partial} \partial \phi_{t}\right)^{n-1}}{\left(\chi_{0}+i \bar{\partial} \partial \phi_{t}\right)^{n}},
$$

which is parabolic. We arrive here at the point of view explained in [3]. We interpret the set $\mathcal{H}_{0}$ of Kahler forms cohomologous to $\chi_{0}$ as the images of the fixed form $\rho$ under the maps in a single complexified orbit in $\mathcal{M}$. So the problem of finding a zero of the moment map in a complexified orbit leads us to the following

Problem 16. Let $(M, \omega)$ be a Kahler manifold and let $\left[\chi_{0}\right]$ be another Kahler metric on $M$. Can one find a Kahler metric $\chi$ in the cohomology class $\left[\chi_{0}\right]$ such that

$$
\chi^{n-1} \wedge \omega=c \chi^{n}
$$

where $c$ is a constant?

The equation (17) is a nonlinear elliptic PDE of Monge-Ampere type for the Kahler potential of $\chi$. If the cohomology class of $\chi_{0}$ is the same as $[\omega]$ there is a trivial solution, $\chi=\omega$, and for nearby cohomology classes one can use a the implicit function theorem to find a solution. Moreover an argument using the maximum principle shows that if a solution exists it is unique. However, the problem does not always have a solution. To see this, notice first that if a solution does exist then the constant $c$ is determined topologically by the cohomology classes of the forms. Now consider a solution of (17) at a fixed point in $M$. We can choose an basis for the tangent space at this point which is orthonormal with respect to $\omega$ and in which $\chi$ is diagonal, with diagonal entries $\lambda_{i}$. The condition is:

$$
\sum \lambda_{i}^{-1}=n c
$$


so $n c \lambda_{i} \geq 1$ for each $i$. This means that $n c \chi-\omega$ is a positive $(1,1)$ form on $M$. Conversely if $[n c \chi-\omega]$ is not a Kahler class then no solution can exist. For example if $M$ is a complex surface, $n=2$, we may suppose that $[\omega]^{2}=1$ and write

$$
[\chi]=s[\omega]+t \eta,
$$

where $\eta \in H^{2}(M)$ with $\eta^{2}=-1, \eta \cdot[\omega]=0$. Then the necessary condition is that:

$$
2 c[\chi]-[\omega]=\left(s^{2}-t^{2}\right)^{-1}\left(\left(s^{2}+t^{2}\right)[\omega]+2 s t \eta\right)>0 .
$$

Now let $R$ be the supremum of the set of parameters $r$ such that $[\omega]+r \eta$ is a Kahler class, and suppose that $R<1$. Then taking $\chi=[\omega]+t \eta$ where $t$ is slightly less than than $R$, we find that $2 c[\chi]-[\omega]$ is not a Kahler class. Thus we conclude that if (16) has a solution for all Kahler classes on a complex surface $M$ then the Kahler cone of $M$ is a component of the entire positive cone for the intersection form i.e. the Kahler cone consists of the $(1,1)$ classes $\chi$ with $\chi^{2}>0, \chi \cdot \omega>0$. By the Nakai criterion this is true precisely when $M$ does not contain any curves of negative self-intersection. Of course the obvious conjecture is that this necessary condition, $[n c \chi]-[\omega]>0$, is also sufficient for the existence of a solution to (16). One would expect that in cases when this condition is violated the solution to the parabolic equation (15) will blow up over some curves of negative self-intersection.

4.4. Surfaces in 4-manifolds. Here we will discuss cases when the maps involved are immersions, rather than diffeomorphisms. We suppose that $M$ is a complex symplectic manifold of complex dimension $2 m$, with a holomorphic symplectic form $\Theta$, and consider the space $\mathcal{N}$ of maps $f$ from our symplectic $2 m$ manifold $S$ to $M$ with $f^{*}(\Theta)=\rho$. We consider the moment map gradient equation

$$
\frac{d f}{d t}=I f_{*}\left(\xi_{f}\right)
$$

where $\xi_{f}$ is the Hamiltonian vector field of the function $\omega \wedge \rho^{m-1} / \rho^{m}$ on $S$, with respect to the fixed symplectic form $\rho$. This evolution preserves $\mathcal{N}$, by the discussion of (3.2). We may regard the equation as an evolution equation for a 1-parameter family of (immersed) LS submanifolds $P_{t} \subset M$ : instantaneously the normal velocity of $P_{t}$ is the normal component of $I X$ where $X$ is the vector field on $P_{t}$ obtained as the Hamiltonian vector field on the symplectic manifold $\left(P_{t},\left.\Theta\right|_{P_{t}}\right)$. Thought of in this way, the equation (18) becomes parabolic, so short-time solutions exist and one might hope to prove that solutions exist for all time and converge to zeros of the moment map: i.e. to submanifolds satisfying (7).

Now let us suppose that $M$ has real dimension 4 and is hyperkahler, as in (3.3). Then the potential limits of the flow (18) on the LS surfaces in $M$ are complex curves for an appropriate complex structure. Let us consider, more generally, the hyperkahler picture. We return to the full space $\mathcal{M}$ of maps from $S$ to $M$ on which we have three moment maps $\mu_{1}, \mu_{2}, \mu_{3}$ for the action of $\mathcal{G}^{\mathrm{Sp}}$. In line with the general theory, we consider the functional

$$
E(f)=\left\|\mu_{1}\right\|^{2}+\left\|\mu_{2}\right\|^{2}+\left\|\mu_{3}\right\|^{2},
$$

on the space $\mathcal{M}$. The gradient flow of this functional is

$$
\frac{d f}{d t}=I f_{*}\left(\xi_{1}\right)+J f_{*}\left(\xi_{2}\right)+K f_{*}\left(\xi_{3}\right) .
$$


Here the moment maps are functions on $S$ :

$$
H_{i}=f^{*}\left(\omega_{i}\right) / \rho,
$$

and $\xi_{i}$ is the Hamiltonian vector field of $H_{i}$ on $S$. So, when restricted to the set of maps $\mathcal{N}$, this hyperkahler flow coincides with the flow (18) we considered before since $H_{2}, H_{3}$ are constants on $S$ in this case, and $\xi_{2}, \xi_{3}$ vanish. To clarify the geometrical meaning of (19), recall that the Grassmannian of oriented 2-planes in a tangent space $T M_{p}$ can be identified with a product of 2 -spheres:

$$
G r_{2}=S\left(\Lambda_{+}^{2}\right) \times S\left(\Lambda_{-}^{2}\right)
$$

and the three Kahler forms $\omega_{1}, \omega_{2}, \omega_{3}$ provide a standard orthnormal basis for $\Lambda_{+}^{2}$. So if $p$ lies in the image of a map $f$ and if we write $\lambda^{2}=H_{1}^{2}(p)+H_{2}^{2}(p)+H_{3}^{2}(p)$ then $H_{i} / \lambda$ are the three standard co-ordinates specifying the $S\left(\Lambda_{+}^{2}\right)$-component $T f(S)_{p}^{+}$of the tangent space $T f(S)_{p} \in G r_{2}$. The Riemannian area form $d A$ induced on $S$ from the metric on $M$ is simply $d A=\lambda \rho$. So the total area of the image surface is

$$
\operatorname{Area}(f(S))=\int_{S} \lambda \rho
$$

while the hyperkahler energy is

$$
E(f)=\int_{S} \lambda^{2} \rho .
$$

(More precisely, the energy functional as we have defined it is given by the sum of $L^{2}$ norms of the functions $H_{i}-\hat{H}_{i}$, where $\hat{H}_{i}$ are their average values, determined by the homotopy class of the map. This is because the constant Hamiltonians act trivially. However the two functionals differ by a constant.) Comparing the two integrals we see that

$$
\operatorname{Area}(f(S)) \leq \sqrt{I} \sqrt{E}(f)
$$

where $I$ is the integral of $\rho$, a constant in the problem. Equality holds in (20) if and only if $\lambda$ is a constant. The relation between area and hyperkahler energy is thus much the same as the familar relation between length and energy of paths in a Riemannian manifold. In particular we have an immediate corollary

Proposition 21. An immersion $f: S \rightarrow M$ is a critical point of the energy functional $E$ if and only if its image is a minimal surface in the ordinary sense and the Riemannian area $d A$ is a constant multiple of $\rho$.

In view of this, it is not surprising that the flow (19) is related to the meancurvature flow studied in Riemannian geometry. To see this, consider an immersion $f: S \rightarrow M$ and the resulting "Gauss map" $\gamma_{f}: S \rightarrow S^{2}$, given by $\gamma_{f}(x)=T f(S)_{f(x)}^{+}$. Suppose, without loss of generality, that at a given point $x \in S, \gamma(x)=\omega_{1}$, so there is a preferred complex structure $I$ on $T M_{f(x)}$, which has the property that tangent space $T f\left(S_{x}\right)$ is a complex subspace. So we have an induced complex structure on $T S_{x}$. The derivative of the Gauss map $\gamma_{f}$ at $x$ is a $\mathbf{R}$-linear map $D: T S_{x} \rightarrow T S_{\omega_{1}}^{2}=\mathbf{R} \omega_{2}+\mathbf{R} \omega_{3}$. We can decompose $D$ into a sum of a complex linear and complex anti-linear part $D=D^{\prime}+D^{\prime \prime}$, using the standard complex structure on $S^{2}$ and the complex structure induced by $I$ on $T S_{x}$. Some calculation shows that mean curvature $h$ of the image surface $f(S)$ at $f(x)$ can be identified with $D^{\prime}$ when we use the natural identification 
of the normal bundle of $f(S)$ at $f(x)$

$$
\nu_{f(S)} \cong T S^{*} \otimes_{\mathbf{C}} T S_{\omega_{1}}^{2} .
$$

Using this point of view one sees that the deformation vector field $I \xi_{1}+J \xi_{2}+K \xi_{3}$ can be decomposed into components tangential and normal to the surface $f(S)$ where the normal component is $\lambda h$ and the tangential component is the gradient in the ordinary Riemannian sense of the function $\lambda$ on $f(S)$. So we may think of our flow as generating a 1-parameter family of pairs $\left(P_{t}, \lambda_{t}\right)$, where $P_{t}$ is an immersed surface and $\lambda_{t}$ is a positive function on $P_{t}$ : instantaneously $P_{t}$ evolves by the mean curvature vector, scaled by $\lambda$, while $\lambda$ evolves by a variant of the equation studied in (4.1) above.

We may apply our general discussion from (2.3) of the stability of non-minimal critical points of the norm of the hyperkahler moment map in this case. A critical point is a parametrised minimal surface, and it is easy to see from the relation between energy and area that this index is the same as the usual index in minimal surface theory. The relevant linear operator is a map:

$$
D: C^{\infty}(S)_{0} \otimes \mathbf{R}^{4} \rightarrow \Gamma\left(f^{*} T M\right),
$$

where $C_{0}^{\infty}$ denotes the functions of integral zero. This is simply

$$
D\left(h_{0}, h_{1}, h_{2}, h_{3}\right)=f_{*}\left(\xi_{0}\right)+I f_{*}\left(\xi_{1}\right)+J f_{*}\left(\xi_{2}\right)+K f_{*}\left(\xi_{3}\right),
$$

where $\xi_{i}$ is the Hamiltonian vector field of the function $h_{i}$ on the symplectic surface $(S, \rho)$. This is an elliptic operator, in fact the symbol is the same as that of the Cauchy-Riemann operator on $\Sigma$ with values in a vector bundle

$$
V=\underline{\mathbf{C}} \oplus T \Sigma^{*} \otimes_{\mathbf{C}} \nu_{f}^{*},
$$

where $\nu_{f}$ is the normal bundle of the immersion, regarded as a complex line bundle. The index of this Cauchy-Riemann operator in the ordinary sense, but taking real dimensions, is

$$
2\left(c_{1}(V)+2(1-g)\right)=-2 e,
$$

where $e$ is the Euler number of the normal bundle. The index of $D$ is thus $-2 \mathrm{e}-4$, where we substract 4 for the constants $C^{\infty} / C_{0}^{\infty}$. Hence the index $d$ in our problem is $d=(2 e+4)$. We obtain, from the general hyperkahler theory (Proposition 6), a result which is related to a theorem of Micallef and Wolfson [9]

Proposition 26. Any compact, immersed, minimal surface in a hyperkahler 4manifold with normal Euler number $e \geq-2$, and which is not a complex curve for some complex structure on $M$, is not strictly stable.

Finally, we point out that although we have derived the energy functional and evolution equation from the hyperkahler point of view the formulae above show that they can be defined for maps from a surface into any oriented Riemannian 4-manifold. This is rather similar to the case of Yang-Mills theory on a 4-manifold: when the manifold is hyperkahler the Yang-Mills functional can be viewed as the norm of the hyperkahler moment map, but the functional makes sense for general manifolds.

4.5. Symplectic forms on 4-manifolds. We will now discuss a hyperkahler version of the case studied in (4.3), so we let $M$ be a (compact) hyperkahler 4-manifold and suppose that $S$ is a symplectic 4-manifold diffeomorphic to $M$. For simplicity we suppose that $\rho$ is cohomologous to $f^{*}\left(\omega_{1}\right)$. We consider the set $\mathcal{M}$ of diffeomorphisms from $S$ to $M$. This is hyperkahler, with a hyperkahler moment map for the action of the symplectomorphism group $\mathcal{G}^{\mathrm{Sp}}$. On $\mathcal{M}$ we have an energy functional $E$ in the 
scheme of (2.3). As before this can be expressed in terms of the induced symplectic form $\chi=\left(f^{*}\right)^{-1}(\rho)$ on $M$. If we write $\chi \cdot \omega_{i}=g_{i}$, using the standard inner product given by the fixed metric on $M$, then the energy is given by:

$$
E(\chi)=\int_{M} \frac{g_{1}^{2}+g_{2}^{2}+g_{3}^{2}}{|\chi \wedge \chi|} d V
$$

where $d V$ is the standard Riemannian volume form on $M$. That is

$$
E(\chi)=\int_{M} \frac{\left|\chi_{+}\right|^{2}}{|\chi \wedge \chi|} d V
$$

Here, as usual, $\chi_{+}$is the self-dual part of $\chi$ with respect to the fixed metric on $M$. The absolute minimum of $E$ is attained when $\chi=\omega$, and we know that any higher critical points cannot be strictly stable, since one readily sees that the relevant index $d$ is 0 . The hyperkahler gradient equation goes over to a certain parabolic evolution equation on the space of symplectic forms on $M$. This suggests the possibility of applications to symplectic topology. Let $\mathcal{S}$ be the space of symplectic forms on $M$, in the cohomology class $\left[\omega_{1}\right]$. A priori this could be disconncted, i.e. there could be different deformation classes of symplectic structure. If one could build up a calculus of variations for the energy functional $E$ on $\mathcal{S}$ one would hope to show that each connected component contains a local minimum of $E$. But we know that the only strictly stable critical point is the standard structure $\omega_{1}$, suggesting that in fact $\mathcal{S}$ should be connected.

Of course there are a great many ways in which this programme could fail: one would certainly need to consider critical points "at infinity" in $\mathcal{S}$. Let us just observe that the energy functional does give some control of the symplectic form. If we write

$$
\left|\chi_{+}\right|=|\chi \wedge \chi|^{1 / 2} \frac{\left|\chi_{+}\right|}{|\chi \wedge \chi|^{1 / 2}}
$$

we obtain

$$
\begin{aligned}
\int_{M}\left|\chi_{+}\right| d V & \leq\left(\int_{M}|\chi \wedge \chi| d V \cdot \int_{M} \frac{|\chi+|^{2}}{|\chi \wedge \chi|} d V\right)^{1 / 2} \\
& =\left(\int_{M} \chi \wedge \chi \cdot E(\chi)\right)^{1 / 2} \\
& =\left(\left[\omega_{1}\right]^{2} E(\chi)^{1 / 2} .\right.
\end{aligned}
$$

On the other hand $\left|\chi_{-}\right|<\left|\chi_{+}\right|$pointwise, since $\chi \wedge \chi>0$. So we deduce that the $L^{1}$ norm of $\chi$ is bounded by a fixed multiple of $E(\chi)^{1 / 2}$. Thus any minimising sequence for $E$ has a subsequence which converges weakly, to some closed current on $M$.

Notice that again the final expression (28) for the functional $E$ does not involve the hyperkahler structure explicitly, so one can try to extend at least some of the ideas to more general 4-manifolds.

\section{REFERENCES}

[1] M. F. AtiYAh AND R. BotT, The Yang-Mills equations over Riemann surfaces, Philosophical Trans. Roy. Soc. London, Series A, 308 (1982), pp. 523-615.

[2] S. K. Donaldson, Remarks on gauge theory, complex geometry and 4-manifold topology, in The Field's Medal Volume, World Scientific, 1998, pp. 384-403. 
[3] S. K. Donaldson, Symmetric spaces, Kahler geometry and Hamiltonian dynamics, in Proc. Northern California Seminar on Symplectic Geometry, Weinstein and Eliashberg, ed., To appear.

[4] M. Gross, Special Lagrangian fibrations II: Geometry, Preprint.

[5] R. Harvey and H. B. Lawson, Calibrated geometries, Acta Mathematica, 148 (1982), pp. 47157.

[6] N. J. Hitchin, The moduli space of special Lagrangian submanifolds, Preprint (dg-ga/9711002).

[7] N. J. Hitchin, A. Karlhede, U. Lindstrom, And M. RoceK, Hyperkahler metrics and supersymmetry, Communications in Math. Physics, 108 (1987), pp. 535-89.

[8] F. C. KIRWAN, Cohomology of Quotients in Symplectic and Algebraic Geometry, Princeton U.P., 1984.

[9] M. J. Micallef AND J. WOLFSON, The second variation of area of minimal surfaces in 4manifolds, Mathematische Annalen, 295 (1993), pp. 245-67.

[10] A. Strominger, S. T. Yau, And F. ZAslow, Mirror symmetry is T-duality, Nucl. Phys., B 479 (1996), pp. 243-59.

[11] C. H. Taubes, Stability in Yang-Mills theories, Communications in Math. Physics, 91 (1983), pp. 235-263. 
S. K. DONALDSON 